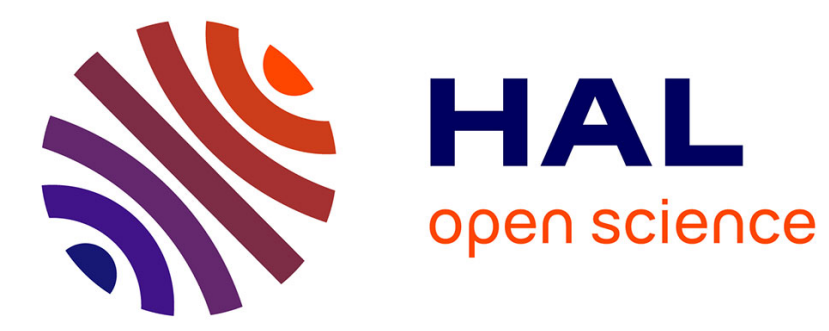

\title{
Mixing Global and Local Competition in Genetic Optimization based Design Space Exploration of Analog Circuits
}

\author{
Abhishek Somani, P. P. Chakrabarti, Amit Patra
}

\section{- To cite this version:}

Abhishek Somani, P. P. Chakrabarti, Amit Patra. Mixing Global and Local Competition in Genetic Optimization based Design Space Exploration of Analog Circuits. DATE'05, Mar 2005, Munich, Germany. pp.1064-1069. hal-00181272

\section{HAL Id: hal-00181272 \\ https://hal.science/hal-00181272}

Submitted on 23 Oct 2007

HAL is a multi-disciplinary open access archive for the deposit and dissemination of scientific research documents, whether they are published or not. The documents may come from teaching and research institutions in France or abroad, or from public or private research centers.
L'archive ouverte pluridisciplinaire HAL, est destinée au dépôt et à la diffusion de documents scientifiques de niveau recherche, publiés ou non, émanant des établissements d'enseignement et de recherche français ou étrangers, des laboratoires publics ou privés. 


\title{
Mixing Global and Local competition in Genetic Optimization based Design Space Exploration of Analog circuits
}

\author{
Abhishek Somani, P. P. Chakrabarti, Amit Patra \\ Advanced VLSI Design Lab., IIT Kharagpur, India \\ somani@vlsi.iitkgp.ernet.in,ppchak@cse.iitkgp.ernet.in, amit@ee.iitkgp.ernet.in
}

\begin{abstract}
The knowledge of optimal design space boundaries of component circuits can be extremely useful in making good subsystem-level design decisions which are aware of the parasitics and other second-order circuit-level details. However, direct application of popular Multi-objective genetic optimization algorithms were found to produce Pareto fronts with poor diversity for analog circuits problems. This work proposes a novel approach to control the diversity of solutions by paritioning the solution space, using Local Competition to promote diversity and Global competition for convergence, and by controlling the proportion of these two mechanisms by a Simulated Annealing based formulation. The algorithm was applied to extract numerical results on analog switched capacitor integrator circuits with a wide range of tight specifications. The results were found to be significantly better than traditional GA based uncontrolled optimization methods.
\end{abstract}

\section{Introduction}

Including an optimization framework in the design of analog circuits has become fairly important in recent times. Typical analog circuit sizing optimization problems require handling of multiple, usually non-commensurate and often conflicting cost criteria. Earlier efforts in this area focussed on methodologies which converted some of the design objectives into constraints and combined the remaining into a single objective function[1]. However, comparatively newer analog systems design methodologies[2] require design of component circuits with optimal design surfaces instead of an optimal design point. At the circuit level, this translates into a problem of optimization to obtain parameterized behavior of circuits. An example of such a parameter is the load capacitance for analog components in MOS integrated analog systems. This necessitates a methodology for generating multiple optimal solutions for the circuits in the entire range of the parameterized variables/specifications. Traditionally, multi-objective optimization methods try to address this problem of generating near-optimal, non-dominated design points (Pareto Front) that are well distributed over the design space[3][4][5][6].

One method of solving a multi-objective circuit optimization problem is to transform it into a set of scalarized single objective optimization problems by the weighted sum approach or the Normal-Boundary Intersection method[4]. However, methods applying deterministic algorithms like steepest descent or quadratic programming for solving the single objective optimization problems have been known to get trapped in local optima. Recently, application of sequential stochastic refinement methods like Genetic Algorithms(GA) for analog circuits design space exploration have been successfully reported in [5][6]. The population based approach of GA makes it very suitable for multiobjective optimization problems, where multiple solutions are sought in a single run of the algorithm. This work explores the possibility of obtaining near-optimal design surfaces using GA methods for analog circuits. An example analog circuit is described in Section 2. Results obtained by application of known GA approaches to this problem are presented in Section 3. We then propose a variation based on mixture of local and global competition in GA and present the associated results in Section 4. For the sake of clarity of expression and explanation, these algorithms have been described for two-objective problems. The extension to an arbitrary number of objective functions is straightforward. Section 5 concludes the paper with a brief discussion on the results in the previous section.

\section{Integrator Circuit}

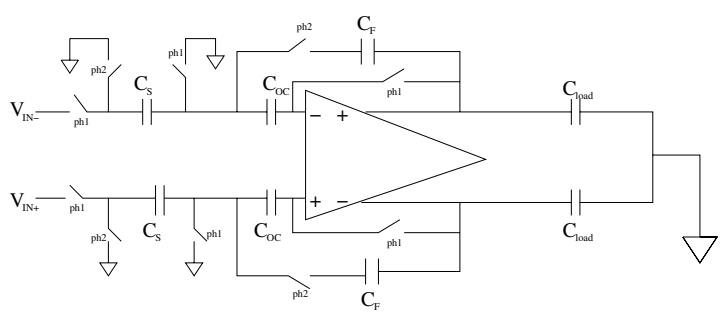

Figure 1. CDS Offset-compensated SwitchedCapacitor Integrator

Our chosen example sub-circuit is a correlated double sampling (CDS) offset-compensated switched-capacitor integrator, which is the basic building block for sigma-delta modulators used extensively to build filters and data converters. We wish to use the optimal design surface of this circuit for the construction of a fourth-order sigma-delta modulator. Fig. 1 shows a typical CDS offset-compensated integrator with sampling, feedback and offset cancellation capacitors in the differential configuration. 
Standard two-stage opAmp topology was used in the integrator for this work. Apart from the gate capacitances of MOSFETs, we consider bottom-plate parasitic capacitances of standard integrated capacitors and drain diffusion and overlap capacitances of MOSFETs for more accurate description and prediction of circuit behaviour. We combine velocity saturation effect and advanced mobility models to arrive at the MOSFET model (shown in eqn.1) for deep submicron MOS circuit optimization.

$$
I_{D}=\frac{\frac{1}{2} \mu C_{o x} \frac{W}{L}\left(V_{G S}-V_{T}\right)^{2}\left(1-\frac{V_{G S}-V_{T}}{E_{s a t} L}\right)\left(1+\lambda V_{D S}\right)}{1+\theta_{1}\left(V_{G S}+V_{T}-V_{K}\right)^{\frac{1}{3}}+\theta_{2}\left(V_{G S}+V_{T}-V_{K}\right)^{n}}
$$

where $\theta_{1}, \theta_{2}$ and $V_{K}$ are fitting parameters, and the rest of the symbols have their usual meanings. Here, $n=1$ for NMOS and 2 for PMOS. The target technology is an industry-standard $0.18 \mu m, 1.8$ Volts, n-well digital CMOS process.

After initial topology based reduction of design parameters, the optimization problem is framed with 15 design parameters. The two objectives are Power dissipation and the Load capacitance. The other circuit performance parameters, viz., Dynamic Range (DR), Settling Time (ST), Settling error (SE), Area, Output voltage range (OR) and Yield Calculation[6] (Robustness) were fixed as circuit constraints. Further the problem was constrained by the requirement of all the transistors to be in the proper DC operating region and matching constraints across all manufacturing process corners. It may be noted here that the effect of non-dominant poles and zeros was included in the equations for ST, SR and DR, which makes them more non-linear than those obtained by standard dominant pole analysis based derivations. This helps in making the entire search-space available to the optimizer by means of a small number of analytical equations. 20 different specifications of the circuit graded by their level of difficulty were chosen and the Pareto Fronts were obtained for all of them using the algorithms described in the following sections. The results obtained for all of the cases showed some common trends, which we will present with explicit illustrations for only one of them due to space limitations in this paper. The specifications for the chosen case are : DR $\geq 96 \mathrm{~dB}$, OR $\geq 1.4$ volts, $\mathrm{ST} \leq 0.24 \mu \mathrm{sec}, \mathrm{SE} \leq 7 \times 10^{-4}$, Robustness $\geq 0.85$.

\section{Applying known GA approaches}

A popular Multi-objective Genetic optimization algorithm called Elitist non-dominated sorting GA (NSGAII)[3] with a real-parameter continuous space was chosen for approaching the above problem. However, the results obtained were not very encouraging. Fig. 2 shows the Pareto-optimal front obtained after 800 iterations of NSGA-
II. Here, it was desired that the algorithm produce Paretooptimal solutions which are well-distributed over the entire range of Load capacitance from 0 to $5 \mathrm{pF}$, but the solutions were found to cluster mostly between 4 and $5 \mathrm{pF}$.

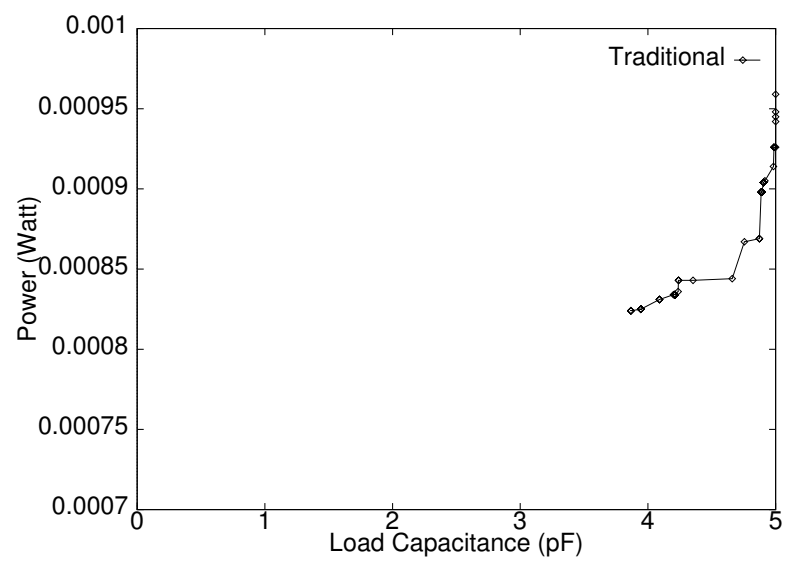

Figure 2. Pareto-optimal Front after 800 iterations of NSGA-II

Investigations into such clustered behavior of the Paretooptimal solutions revealed the following :

- Loss of diversity : If in the early phase of GA, a comparatively higher number of solutions are produced in a small part of the objective function space in comparison to the rest of the space, the "cross-over" operator tends to produce more solutions "in between" the solutions in the cluster and thus tends to make the cluster more dense.

- Premature Termination : Comparatively weaker solutions from the rest of the space are gradually lost due to failure to win in the global competition. This leads to further loss in diversity and ultimately premature termination of evolution.

- Population Size: Clustering effect can be reduced by increasing population size considerably, but this increases the computational cost also.

\section{Partition based Multiple Objectives Opti- mization Methodology}

The core problem identified in the previous section was to introduce mechanisms which can preserve diversity in the population while not sacrificing the speed of convergence to the near-optimal Pareto Front in a resource-efficient manner. A first approach for increasing diversity is to increase the mutation probability. Increased mutation helps upto a certain extent beyond which the entire optimization process becomes random and loses the focus required for convergence in reasonable time. Also, the optimal value of mutation probability is heavily problem-dependent and its estimation is non-trivial. 


\subsection{Contribution of this work}

A known method of diversity preservation is parallel population GA with inter-population migration controlled in a tribe or island based framework[7], which can be extended for Multi-objective GA. However, in this work, we try to establish that this objective can be accomplished by a simple modification in the traditional single-population GA. We argue that if the relatively weaker solutions from sparsely populated regions of the objective function space can be protected from global competition and evolved until they become strong enough to compete globally, one would get reasonably diverse solutions in the final Pareto optimal front. To achieve this, a Simulated Annealing driven Competition in Genetic Algorithm (SACGA) is proposed in this paper, where the objective function space is partitioned and a purely Local Competition in each partition transforms into a purely Global competition in the entire space in the course of the evolution of the GA. Further, a Multi-phase Expanding-partitions SACGA (MESACGA) is proposed to speed up the rate of advancement of the Pareto Front.

\subsection{Performance Metric}

The performance metric for the results obtained from a Multi-objective Optimization Algorithm should evaluate both the diversity of the solutions and their convergence to the near-optimal Pareto front. One such metric for minimization problems is the Hypervolume Metric[3]. Mathematically, for each solution $i$, a hypercube $v_{i}$ is constructed with the origin of the objective function space and the solution $i$ as the diagonal corners of the hypercube. Thereafter, a union of all hypercubes is found and its hypervolume is calculated. Obviously, a lower value of the hypervolume indicates a better pareto front.

\subsection{Local Competition only}

Given two objective functions, we propose to partition the associated function space into $\mathbf{m}$ equal partitions induced by the division of the range space of any one of the objective functions into $\mathbf{m}$ equal, disjoint partitions. In each iteration, only Local competition is allowed within each partition for non-dominated ranking of individuals. A Global Mating Pool is created by rank-based selection of individuals from the entire population of solutions at the end of each iteration and the candidates for next iteration are created by performing cross-over and mutation operations in the Global Mating Pool. After a fixed number of iterations, Global Competition is performed once on the entire population to find the Global Pareto Front containing the set of globally non-dominated solution points.

This method produces reasonably diverse solutions and keeps the eligible candidates from partitions falling within the feasible objective function space alive for large number of iterations, thus allowing them improved chance to participate in the Global Mating Pool. However, after the initial stage of a couple of hundred iterations, the
Global Pareto Front was found to progress towards the near-optimal Pareto front extremely slowly and would require large number iterations to reach there. The reason for this observation can be better explained by defining a few terms: The population members on the Pareto front of the global population are called Globally Superior solutions and the remaining are termed Globally Inferior solutions. Similarly, the best individuals obtained from $\mathrm{Lo}$ cal competition of individuals in a subset of the population form the Local Pareto Front and are termed Locally Superior solutions. Thus, in a GA, the cross-over operation has a better chance of producing more Globally Superior solutions when operating on two Globally Superior solutions, than when operating on two Globally Inferior solutions or a Globally Superior solution with a Globally Inferior solution. In absence of any Global Competition, there are typically many Locally Superior solutions which are Globally Inferior. This causes slow production of Globally Superior solutions.

\subsection{Mixing Local and Global Competition}

It is evident that while Local competition improves the diversity of the solutions along the Global Pareto Front, Global Competition improves the speed of movement of the front towards the global near-optimal front. We propose a Simulated Annealing driven Competition in Genetic Algorithm (SACGA) to allow the evolution process in the algorithm to gradually transit from pure Local competition in the initial phase to pure Global Competition in the final phase of the duration of solving the problem. Fig.3 shows the flow of this algorithm. The phase of mixed local and global competition is preceded by a phase of pure local competition which ends after each partition has evolved at least one solution which satisfies all the constraints. However, some partitions may be in the infeasible region. So, we define an upper limit on the number of iterations allowed in this phase, after which partitions with no constraintsatisfying solutions are discarded. Let $n$ be the desired number of globally non-dominated (i.e., superior) solutions per partition at the end of the second phase and gen $n_{t}$ be the number of iterations required for the first phase. Let span be the total number of iterations allowed in the second phase.

In any iteration in the second phase, let $m_{p}$ be the number of locally superior solutions in partition $p$ and $c$ be the cost of allowing a locally superior solution to participate in the global competition. Then, we formulate :

$$
c=k_{1} e^{\left(k_{2} \frac{i}{n-1}\right)}
$$

where, $k_{1}, k_{2}$ are constants to be chosen for shaping the cost functions and $i$ assumes the values $1,2, \ldots, m_{p}$ for partition $p$. Thus, the locally superior solution $i$ will participate in 


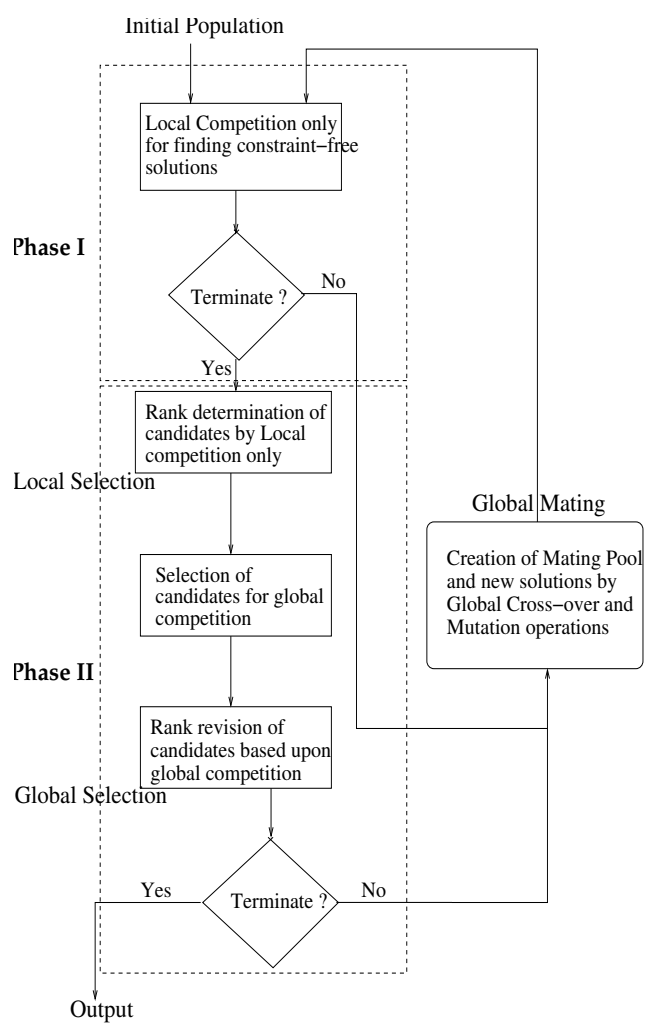

Figure 3. SACGA Algorithm flow

global competition with probability prob given by :

$$
\text { prob }=1-e^{\left(\frac{-\alpha}{c \times T_{A}}\right)}
$$

where $\alpha$ is a chosen constant and Annealing temperature $T_{A}$ is given by the temperature schedule :

$$
T_{A}=T_{i n i t} e^{\left(-k_{3} \times \frac{\ln \left(T_{i n i t}\right)}{s p a n} \times\left(g e n-g e n_{t}\right)\right)}
$$

where, gen is the current iteration number and $T_{A}$ is cooled down from $T_{\text {init }}$ to a value of 1 .

This formulation of probability prob provides the following features :

1. It encourages pure local competition in the initial iterations and pure global competion in the final iterations of this phase.

2. The more the number of locally superior solutions produced by a partition, the better the partition is. However, we do not allow all such "good" solutions to participate in the Global Competition till the very final phase of the evolution. This is necessary because if some of these solutions are retained, then the partition will maintain its representation in the Global pool even if all its participants in the Global Competition are dominated by solutions from other partitions. This is accomplished by assigning different probabilities to different locally superior solutions. Thus, if the $m_{p}$ "good" solutions in a partition $p$ are considered in a randomly determined sequence for evaluation of their candidature for global competition, the solutions considered earlier have a higher probability than those considered later.

3. The shapes of the probability curves can be easily controlled by selecting the parameters $k_{1}, k_{2}$ and $k_{3}$ for desired values of probability at iteration gen $=$ gen $_{t}+\frac{\text { span }}{2}$ for $i=1, n$ and that at iteration gen $=$ $g_{e n}+\operatorname{span}$ for $i=1, \ldots, n$. Fig. 4 shows the probability curves for $n=5$ and $\operatorname{span}=100$.

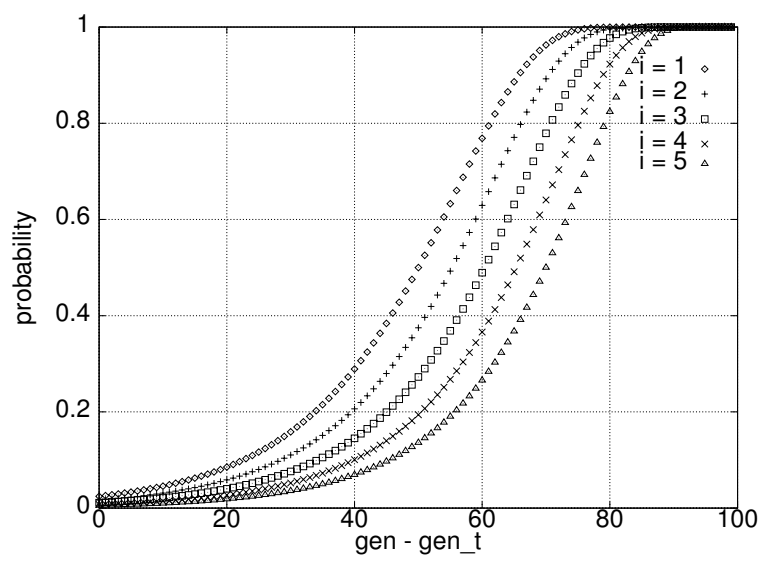

Figure 4. Probability curves for $n=5$

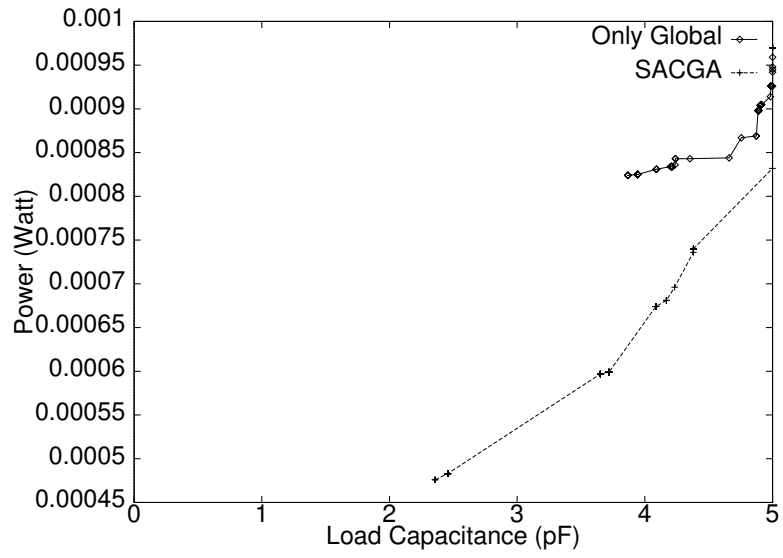

Figure 5. Pareto Fronts after 800 iterations of $i$ ) Traditional Purely Global competition based and $i i$ ) SACGA based evolution

In the example circuit problem, the objective function space partitioning is induced by the division of the range space of the Load Capacitance. Fig.5 presents the Pareto Front obtained after 800 iterations of an 8-partition SACGA and its comparison with that obtained earlier by NSGA-II using purely global competition. A prominent issue which affects the efficiency of SACGA is the problem of selecting the optimal number of partitions with respect to each objective function and determining their (generally, 


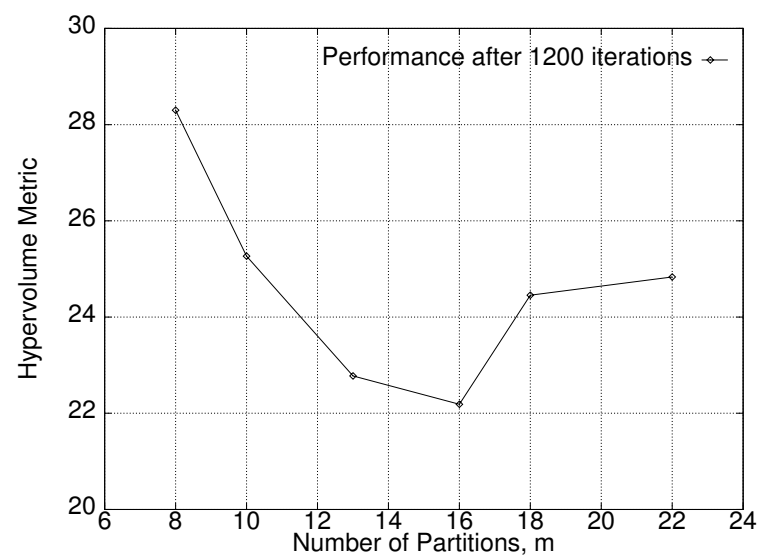

Figure 6. Determination of Optimal number of partitions

unequal) sizes. They are dependent upon the solution space and no method is known of finding them. A simplified approach may be to choose partitions of equal sizes. However, it is intuitive and our studies have shown that the optimality of the solutions obtained by a finite number of iterations depends upon the chosen number of partitions. Fig.6 shows the quality of results obtained after 1200 iterations for the example problem. Dividing the objective function space into 16 equal partitions was found to be optimal for this problem instance. At present, no alternative to complete experimentation is known for finding this number. It is to circumvent this problem coupled with a few other advantages that we suggest a modified version of SACGA in the next subsection.

\subsection{Multi-phase Expanding-partitions SACGA (MESACGA)}

A better option to chosing a set of static partitions would be to start with a high number of partitions and gradually expand the partition-size, thereby reducing their number. Such a method would essentially "grow" the individual Local Pareto Fronts till they combine together to form the Global Pareto Front in the final phase having just one partition covering the entire objective function space. Essentially, MESACGA is a SACGA running in multiple phases where the number of partitions is reduced and their size increased at the end of each phase. A brief overview is shown in Fig.7. MESACGA provides a systematic method for striking a balance between preserving Pareto front diversity and convergence to global near-optimal. In MESACGA, at the end of each phase of SACGA, the partitions start to cover a wider region in the objective function space in the current phase as compared to that in the previous phase. This causes some Locally Superior but Globally Inferior solutions to be thrown away and there is a tendency of decrease in the proportion of Globally Inferior solutions in the pool of Locally Superior solutions and hence, faster movement of the pareto front. However, the presence of such solutions over periods of iterations is necessary for preserv- ing diversity. The parameter span, i.e., the number of iterations for each phase of MESACGA provides a direct control to maintain the above mentioned balance. Thus, a smaller value of span would give quick results which may not cover a significant portion of the objective function space, whereas, a higher value of span would make the conversion of Local Pareto Fronts to the Global Pareto Front more gradual and would, in general, produce solutions with higher diversity. In addition, MESACGA was found to produce results comparable in quality to those produced by selecting the best possible number of equal partitions in SACGA.

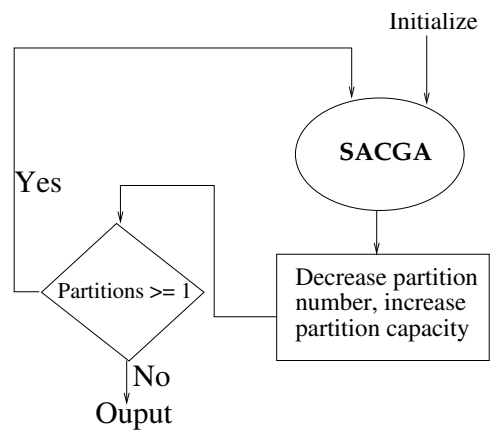

Figure 7. MESACGA Algorithm Flow

MESACGA was applied to the example problem with 7 successive phases of SACGA containing 20,13, 8, 5, 3, 2, 1 partitions respectively. Fig. 8 shows the MESACGA results in comparison to the both traditional and SACGA approaches.

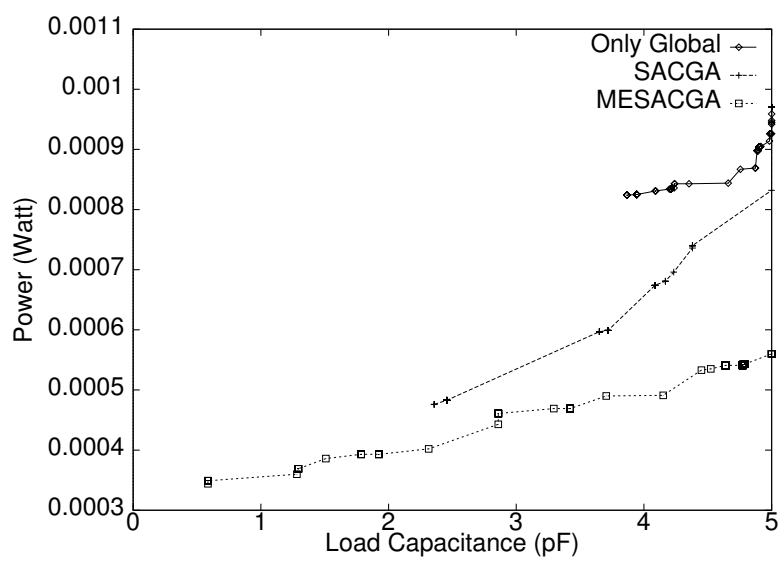

Figure 8. Pareto Fronts obtained after 800 iterations of $i$ ) Purely Global competiton based, ii) SACGA based, and iii) MESACGA based evolution

\section{Discussions and Conclusion}

The results obtained for all the 20 different circuit specifications showed similar trends with both SACGA and MESACGA, taking, on an average, $18 \%$ more computa- 
tional time compared to NSGA-II, due to additional overheads of these algorithms. In brief, the trends were as follows :

1. In all cases, where the evolution was continued for more than 650 iterations, the quality of the solutions obtained for equal number of iterations for TPG (Traditional Purely Global competition based GA), SACGA and MESACGA were found to be in the order MESACGA $\geq$ SACGA $\geq$ TPG. Fig. 8 shows the Pareto Fronts obtained for 800 iterations from the three approaches. However, no clear trends were visible for lesser number of iterations.

2. It was observed in SACGA, that the differential gain obtained by increasing the number of iterations beyond 700 turned out to be very low. Fig.9 plots the Hypervolume metric calculated for the Pareto fronts obtained by setting the span parameter to progressively increasing values in an 8 -partition SACGA. It is clear that not much improvement of the Pareto front is obtained for $\operatorname{span}>1000$.

3. Fig.10 shows the Hypervolume metric calculated for the Pareto fronts at the end of each phase of SACGA in a 7 -phase MESACGA. The value of span was kept the same for each phase and the results were found to improve by increasing the value of span. It was shown in Fig. 6 that a 1200 iteration SACGA produces best results for 16 partitions. It can be clearly observed in Fig. 11 that a 1250 iteration MESACGA having a purely local competition phase of 200 iterations followed by 7 phases of 150 iterations each produces comparable results. The Hypervolume metric calculated for the Pareto fronts are 22.19 and 21.83 for SACGA and MESACGA respectively.

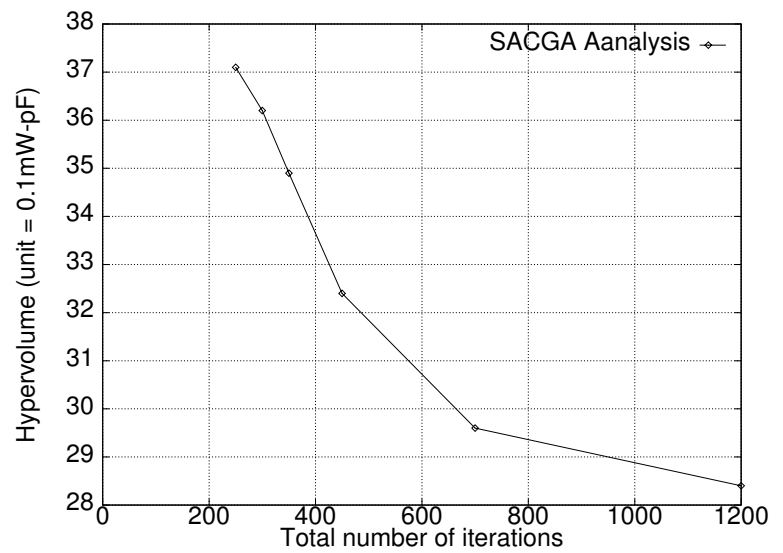

Figure 9. Performance of SACGA for various preset values of total number of iterations

Thus, we conclude that the poor diversity of the Pareto fronts obtained from application of traditional, purely global competition based Multi-objective genetic optimization algorithms can be remedied to a large extent by the proposed MESACGA approach.

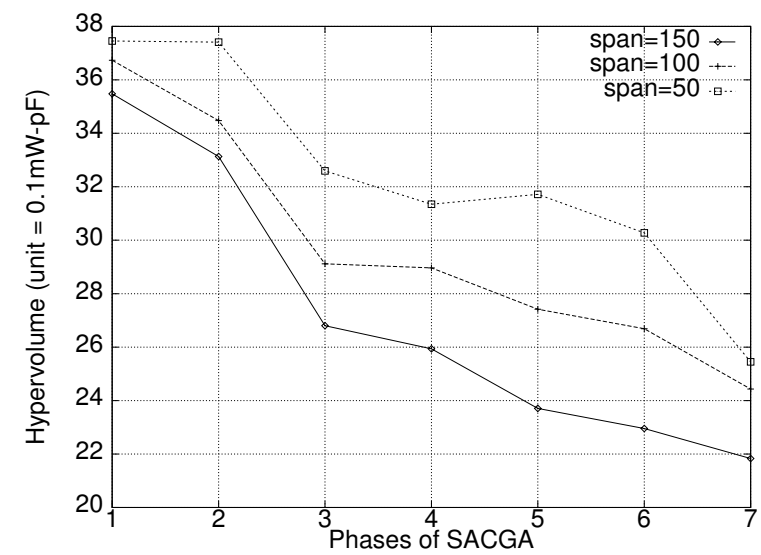

Figure 10. Progress of Pareto Front across various SACGA phases of MESACGA

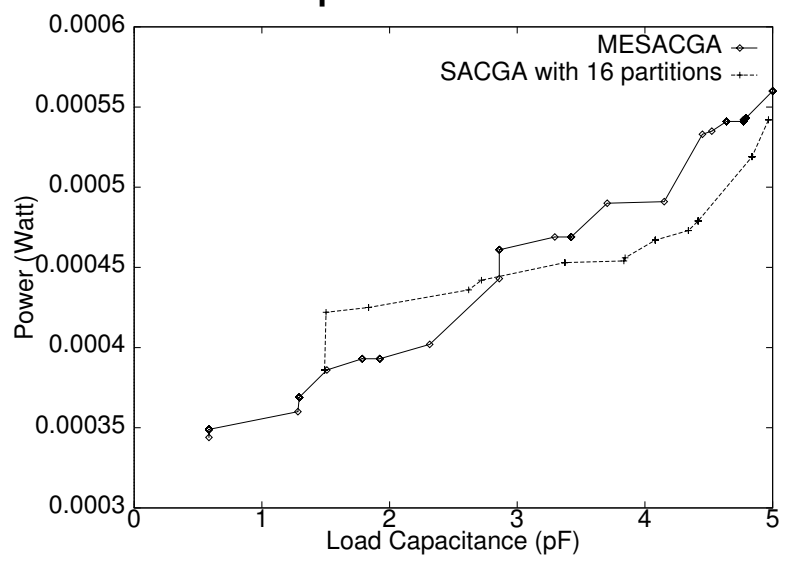

Figure 11. Comparison of MESACGA performance with best SACGA performance

\section{References}

[1] G.Alpaydin, S.Balkir, and G.Düdar. An evolutionary approach to automatic synthesis of high-performance analog integrated circuits. IEEE Tran. Evol. Comp., 7:240-252, June 2003.

[2] F. D. Bernardinis and A. S. Vincentelli. A methodology for system-level analog design space exploration. Design Automation and Test in Europe, 2004.

[3] K.Deb. Multi-Objective Optimization using Evolutionary Algorithms. John Wiley and Sons, Ltd., 2003.

[4] G. Stehr, H. Graeb, and K. Antreich. Performance trade-off analysis of analog circuits by normal-boundary intersection. Design Automation Conference, 2003.

[5] D. Smedt and G.Gielen. Watson: Design space boundary exploration and model generation for analog and RFIC design. IEEE Trans. Computer-Aided Design, 22:213-224, Feb. 2003.

[6] D. Smedt and G.Gielen. Holmes: Capturing the yield optimized design space boundaries of analog and RF integrated circuits. Design Automation and Test in Europe, 2003.

[7] H. Homayounfar, S. Areibi, and F. Wang. An island-based GA for dynamic optimization problems. The 2nd Annual McMaster Optimization Conference: Theory and Applications, 2002. 